\title{
Creencias epistemológicas de docentes de matemáticas en formación y en ejercicio sobre las matemáticas, su enseñanza y aprendizaje*
}

Epistemological Beliefs of Pre-Service and In-Service Math Teachers, Their Teaching and Learning

Crenças epistemológicas de professores de matemáticas em formação e em exercício sobre as matemáticas, seu ensino e aprendizagem

\section{Grace Judith Vesga-Bravo** (iD orcid.org/0000-0002-9990-360x Mary Falk de Losada*** (iD orcid.org/0000-0002-6380-0481}

\footnotetext{
Artículo de investigación

Revista Colombiana de Educación, N.7 74. Primer semestre de 2018, Bogotá, Colombia

Para citar este artículo: Vesga, G. y de Losada, M. (2018). Creencias epistemológicas de docentes de matemáticas en formación y en ejercicio sobre las matemáticas, su enseñanza y aprendizaje Revista Colombiana de Educación, (74), 243-267.
}

\section{(c) $\underset{\mathrm{BY}}{(1)}(8)$}

* Esta investigación fue auspiciada por la Universidad Antonio Nariño a través del Programa de Formación de Alto Nivel (PFAN).

** Doctora en Educación Matemática. Magister en Ciencias Matemáticas de la Universidad Nacional de Colombia. Docente investigadora Universidad Antonio Nariño. Bogotá, Colombia. Correo electrónico: gvesga@uan.edu.co

*** Magister en la Enseñanza de la Matemática (MA) de Harvard University. Docente Investigadora del Doctora do en Educación Matemática, Universidad Antonio Nariño. Bogotá. Colombia.

Correo electrónico: mariadelosada(a)gmail.com 


\section{Resumen}

En este artículo se presenta el resultado de una investigación que tuvo como objetivo analizar y describir las creencias epistemológicas de seis docentes de matemáticas en formación y tres docentes en ejercicio sobre las matemáticas, su enseñanza y aprendizaje, comprender cómo se estructuraron y cómo se transforman. Se utilizó una metodología con enfoque cualitativo, a través de estudios de casos; los participantes asistieron a cursos orientados a desafiar sus creencias epistemológicas y a promover reflexión al respecto. Se usaron como instrumentos cuestionarios cerrados y entrevistas semiestructuradas. Se pudo identificar que es la experiencia docente intensiva la que permite crear y consolidar creencias epistemológicas, que el pregrado constituye una etapa en la que las creencias epistemológicas formadas con anterioridad en el colegio se están ajustando permanentemente, y que la formación recibida, tanto implícita como explícita, es de difícil y lenta asimilación. También, se pudo establecer que las creencias epistemológicas de docentes en formación y en ejercicio están en constante confrontación Esto posibilita que los programas de formación, inicial o continua, incidan de manera más efectiva y explícita en su construcción o transformación. Por último, se plantean elementos que se deben considerar en los programas de formación, encaminados a lograr creencias de acuerdo con los fines de la educación matemática.

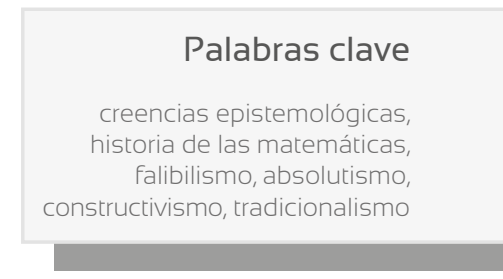

\section{Keywords}

epistemological beliefs; history of mathematics; fallibilism; absolutism; constructivism; traditionalism

\begin{abstract}
This paper presents the results of a research that aimed to analyze and describe the epistemological beliefs of six pre-service math teachers and three in-service math teachers, as well as their teaching and learning, in order to understand how they were structured and how they change. A qualitative approach was used through case studies, where participants attended courses aimed at challenging their epistemological beliefs and encouraging them to reflect on them. The tools used were closed questionnaires and semi-structured interviews. We were able to identify that it is an intensive teaching experience that allows the creation and consolidation of epistemological beliefs, that undergraduate programs are a stage in which the epistemological beliefs previously formed in school are permanently adjusted, and that the training received, both implicit and explicit, is difficult and slow to assimilate. It was also possible to establish that the epistemological beliefs of pre-service and in-service teachers are in constant confrontation. This enables both initial and continuing education programs to have a more effective and explicit influence on their construction or transformation. Finally, we propose a number of elements that should be taken into consideration in training programs, aimed at achieving beliefs that are consistent with the goals of mathematics education
\end{abstract}

\section{Palavras-chave}

crenças epistemológicas; história das matemáticas

falibilismo; absolutismo construtivismo; tradicionalismo 


\section{Introducción}

Las matemáticas cobran cada vez más importancia y se consideran que junto a las ciencias y el lenguaje deben ofrecer el desarrollo de competencias, conocimientos y habilidades necesarias para participar exitosamente en las diferentes actividades y procesos del mundo contemporáneo (Valero, 2017). Esto hace que también cada vez más los diferentes países se preocupen por los resultados de sus estudiantes en diferentes pruebas nacionales e internacionales en estas áreas, en el caso de matemáticas se pueden mencionar el Programa Internacional de Evaluación de Estudiantes (PISA, por sus siglas en inglés), o el Estudio Internacional de Tendencias en Matemáticas y Ciencias (timss, por sus siglas en inglés), en las cuales los estudiantes Colombianos se destacado pero por sus bajos desempeños (ICFES, 2013,2010). Varios factores pueden explicar los bajos resultados de los estudiantes y las diferencias entre ellos, entre los que se ha demostrado la calidad de los docentes como uno de ellos (ICFES, 2010; Yang 2014). En este sentido diferentes investigaciones y organizaciones señala que se puede avanzar en la educación de niños y jóvenes y en el desarrollo de sus competencias matemáticas en la medida en que se formen cada vez mejores maestros. En este sentido cabe preguntarse ¿Qué significa formar docentes de calidad? ¿Qué es útil que los futuros docentes aprendan? O ¿Qué debería enseñarse? Hersh (1997) plantea que la pregunta no es sobre qué matemáticas se debe enseñar sino sobre ¿cuál es la postura epistemológica que se tiene sobre qué son las matemáticas? ¿qué creencias se tienen sobre la matemática y su naturaleza? Porque ésta afecta profundamente la práctica.

La literatura muestra que existe una estrecha relación entre las creencias que tienen los docentes sobre las matemáticas y su enseñanza y aprendizaje, bien sea de manera explícita o implícita (Artz y Armour-Thomas, 1999; Cross, 2009, 2015; Flores, 1995; Pajares, 1992; Penn, 2012; Steiner, 1987; Thompson, 1984, 1992). Además se pone de manifiesto que dichas creencias pueden tener efectos positivos o negativos, por ejemplo frente a la capacidad y disposición de los docentes para probar y desarrollar nuevos enfoques, para incorporar transformaciones en sus prácticas o para lograr que reformas curriculares tengan éxito (Agudelo-Valderrama, 2006; Cross, 2009, 2015; Handal y Herrington, 2003; Pantziara, Karamanou y Philippou, 2013; Pepin, 1999; Steiner 1987). En general, las creencias acerca de la naturaleza de las matemáticas han sido formadas por los docentes a través de modelos de enseñanza que han recibido mucho antes de cursar estudios formales en educación matemática, por lo cual éstos son altamente resistentes al cambio (Cross, 2009). Factores como el entorno escolar y las comunidades a las que pertenecen los docentes son importantes en su construcción y consolidación (Charalambous, Panaoura 
y Philippou, 2009; Chassapis, 2007; White-Fredette 2009). Otras investigaciones indican que no necesariamente existe una alineación entre las creencias que se tienen sobre las matemáticas y la forma en que éstas se enseñan (Penn, 2012).

Estudios señalan que las creencias de los docentes o futuros docentes están altamente influenciadas por los conocimientos filosóficos e históricos que tienen sobre las matemáticas. En este sentido, se pueden encontrar como paradigmas opuestos el absolutismo o formalismo y el falibilismo frente a las creencias sobre la matemática, y el tradicionalismo y el constructivismo en relación con su enseñanza y aprendizaje (Lerman, 1990; Penn, 2012; White-Fredette, 2009/2010). Al respecto Sfard, citada por White-Fredette (2009/2010), señala que, en general, los matemáticos (puros) forman parte del paradigma absolutista. Los investigadores en educación matemática se inclinan más por el falibilista, y los docentes de matemáticas de educación básica y media están atrapados en el medio, con una mayor tendencia al formalismo. La perspectiva falibilista no impregna las creencias acerca de la naturaleza de las matemáticas de futuros profesores (Cooney, Shealy y Arvold, 1998), posiblemente tampoco la de programas de formación. Más recientemente, Gowers (2001) afirma que prevalecen dos culturas en las matemáticas, cuyas raíces responden a cuestiones de énfasis o prioridades y no son mutuamente excluyentes: una en que predomina el entendimiento, la estructura y la demostración, y otra, en la que predomina la solución de problemas.

En el caso colombiano, en uno de los documentos curriculares orientadores, los Estándares básicos de competencias, se afirma que las matemáticas son consideradas "como un cuerpo de prácticas y de realizaciones conceptuales y lingüísticas que surgen ligadas a un contexto cultural e histórico concreto y que están en continua transformación y reconstrucción como otros cuerpos de prácticas y saberes" (MEN, 2006, p. 47). Se plantea allí que para desarrollar competencias matemáticas en los niños y jóvenes es necesario que los docentes, con base en las nuevas tendencias de la filosofía de las matemáticas, adopten un modelo epistemológicamente coherente, lo cual se relaciona con que los docentes reflexionen, exploren y se apropien de supuestos sobre las matemáticas, como

Las matemáticas son una actividad humana inserta en y condicionada por la cultura y por su historia, en la cual se utilizan distintos recursos lingüísticos y expresivos para plantear y solucionar problemas [...]. En la búsqueda de soluciones y respuestas a estos problemas surgen progresivamente técnicas, reglas y sus respectivas justificaciones, las cuales son socialmente decantadas y compartidas.

Las matemáticas son también el resultado acumulado y sucesivamente reorganizado de la actividad de comunidades profesionales [...]. (p. 49). 
Avanzar hacia la consolidación de un modelo epistemológicamente coherente por parte de los docentes de matemáticas requiere que se haga un trabajo continuo que confronte las creencias epistemológicas acerca de las matemáticas y su enseñanza y aprendizaje que genere reflexión alrededor de este saber y sus implicaciones en la práctica (Cross, 2009; Flores, 1995; Roscoe y Sriraman, 2011).

Con base en la descripción anterior, en esta investigación se abordaron como preguntas de investigación:

» ¿Cuáles son las creencias epistemológicas que tienen docentes de matemáticas en formación y en ejercicio acerca de las matemáticas, su enseñanza y aprendizaje, y cómo se transforman al incorporar experiencias de aprendizaje basadas en la filosofía, la epistemología y la historia de las matemáticas?

» ¿Qué elementos deben tenerse en cuenta para realizar cambios significativos en programas de formación de docentes de matemáticas encaminados a que los docentes desarrollen creencias y actitudes más productivas y coherentes hacia las matemáticas, su enseñanza y aprendizaje?

\section{Marco referencial}

Este estudio se ubica en el paradigma de investigación basado en el pensamiento del profesor, y se interesa específicamente por las creencias epistemológicas sobre las matemáticas, su enseñanza y aprendizaje.

\section{Creencias epistemológicas sobre la matemática}

Las creencias epistemológicas han sido conceptualizadas de manera multidisciplinaria, ya que las personas tienen creencias diferentes sobre los distintos aspectos del conocimiento (Buehl y Fives, 2009; Shommer, 1990; Schommer-Aikins, 2002). Basado en los estudios de Shommer, Buehl y Fives (2009) sugieren tres dimensiones para las creencias epistemológicas sobre la matemática: de dónde proviene el conocimiento matemático (fuente u origen); si éste es seguro e inmutable, o tentativo y evolutivo (es decir, estabilidad); y si el conocimiento es simplista y aislado, o complejo e integrado (es decir, estructura). Por otra parte, la literatura muestra que los docentes tienen una amplia gama de creencias sobre las matemáticas que incluso han formado antes de iniciar su educación para ser maestros, están interconectadas con las creencias sobre la enseñanza y el aprendizaje y están altamente influenciadas por los conocimientos filosóficos e históricos que tengan (Charalambous, Panaoura y Philippou, 2009; Chassapis, 2007; Ernest, 1991; Penn, 2012; White-Fredette, 2009). La filosofía de las 
matemáticas tiene como tarea reflexionar acerca de la naturaleza de las matemáticas y dar cuenta de ella a través de preguntas como ¿Cuál es la base para el conocimiento? ¿Cuál es la naturaleza de la verdad matemática? ¿Qué caracteriza las verdades matemáticas?, entre otras (Ernest, 1991). Existe gran variedad de filosofías de la matemática denominadas, entre otras, logicismo, intuicionismo, formalismo, constructivismo, platonismo; en los lineamientos curriculares de matemáticas se señala que

La historia da cuenta de siglos y siglos de diversas posiciones y discusiones sobre el origen y la naturaleza de las matemáticas; es decir, sobre si las matemáticas existen fuera de la mente humana o si son una creación suya; si son exactas e infalibles o si son falibles, corregibles, evolutivas y provistas de significado como las demás ciencias. (MEN, 1998, p. 10).

En los lineamientos se presenta una síntesis de cada una de estas filosofías, y se señala que en el platonismo las matemáticas se ven como un sistema de verdades que siempre han existido y son independientes del ser humano; la tarea que tiene el matemático es descubrir dichas verdades matemáticas. Para el logicismo, las matemáticas son una rama de la lógica; por ello los conceptos matemáticos se deben definir mediante términos lógicos y los teoremas de las matemáticas se deben obtener mediante el empleo de deducciones lógicas. En el formalismo se considera que las matemáticas son una creación de la mente humana, que consta sólo de axiomas, definiciones y teoremas que se obtienen mediante la manipulación de los símbolos y el uso de las reglas establecidas; por tanto, la verdad de las matemáticas está en la mente humana, pero a través del uso coherente de las reglas del juego simbólico establecido. Para el formalista, las demostraciones tienen que ser rigurosas y obtenidas de manera deductiva. En el intuicionismo el principio básico es que sólo existe en las matemáticas lo que en ellas haya sido construido por la mente humana sin menospreciar la intuición. El constructivismo está relacionado con el intuicionismo; ya que considera que las matemáticas son una creación de la mente humana, y sólo tienen existencia real los objetos matemáticos que se pueden construir por procedimientos finitos a partir de objetos primitivos (MEN, 1998).

De otra parte, Lakatos (1976) señala que la multiplicidad de filosofías de las matemáticas se pueden asociar con dos grupos, que llamó euclidiano y cuasi empírico. En el primero, las matemáticas son verdades universales y absolutas, mientras que en el segundo, se ve el crecimiento matemático como un proceso de conjeturas, pruebas y refutaciones. Para Lakatos "el formalismo desconecta la filosofía de las matemáticas de la historia de las matemáticas, puesto que, de acuerdo con la concepción formalista de las matemáticas, éstas no tienen propiamente historia" (p. 17). Putnam (1975) argumenta que el conocimiento matemático se asemeja a un conocimiento empírico, es decir que el criterio de la verdad tanto en las 
matemáticas como en la física es el éxito de nuestras ideas en la práctica, y que el conocimiento matemático es corregible y no absoluto. Hersh (1997) afirma que prevalecen dos tendencias acerca de la naturaleza de las matemáticas: el platonismo y el formalismo, y se manifiesta en contra de las dos; propone crear una nueva, afirmando que las matemáticas son una creación humana, el conocimiento matemático es falible, por tanto las matemáticas pueden avanzar por cometer errores, al corregir sobre ellos. Existen diferentes formas de validar el conocimiento matemático, no solo la aristotélica, y los objetos matemáticos son parte de la cultura.

Estas diferentes posturas filosóficas sobre las matemáticas se pueden dividir en dos grandes miradas que describen creencias epistemológicas acerca de las matemáticas: absolutista y falibilista. Estas se consideran para el desarrollo del presente estudio. En el absolutismo se considera que las matemáticas son verdades universales y absolutas, por tanto son infalibles, incuestionables y no hay lugar para el error; se utiliza un lenguaje formal, $y$, o bien existen aparte en un mundo de ideas puras (platonismo) o en la mente del creador (neoplatonismo) y se descubren, o se crean a partir de sistemas lógico-deductivos (instrumentalistas o formalistas) (Ernest, 1989, 1991, 1998). Desde el absolutismo se considera que las fuentes del conocimiento son de tipo formal (los expertos, los textos, reportes investigativos, entre otros), y se transmiten a través de figuras de autoridad (Penn, 2012). Se cree además que las matemáticas pueden verse como un conjunto de reglas que no cambian, y una vez aprendidas, las personas deben poder aplicarlas para resolver diferentes problemas. En este sentido, Penn (2012) señala que esta creencia puede conducir a que no hay necesidad de repensar los conceptos o buscar nuevos conocimientos. Por último, la estructura del conocimiento matemático desde esta mirada se considera como una disciplina aislada y discreta, estrictamente demarcada y separada de otros ámbitos del conocimiento; la lógica es el único reino seguro para el conocimiento matemático (Ernest, 1991).

En contraste, desde el falibilismo se considera que las matemáticas son producto de la invención humana, pueden fallar, son susceptibles de ser corregidas, nunca se puede considerar más allá de la revisión y corrección, los conceptos pueden sufrir modificaciones a lo largo del tiempo y se comparten significados dentro de una comunidad. Por lo tanto, el conocimiento matemático es corregible y está abierto de manera perpetua a la revisión (Davis, Hersh y Marchisotto, 2012; Ernest, 1991; Hersh, 1997; Lakatos, 1976). En el falibilismo se cree que el conocimiento matemático tiene como origen fuentes informales que son cuerpos internos de información como resultado de experiencias e interacciones sociales individuales (Buehl y Fives, 2009). En este paradigma se cree que las reglas se generan, y las matemáticas son inventadas y reinventadas. Esto podría conducir a pensar que aprender algo nuevo es una pérdida de tiempo, 
debido a un cambio constante (Penn, 2012). En este paradigma se cree que las matemáticas están conectadas indisolublemente como parte de todo el tejido del conocimiento humano (Ernest, 1991).

\section{Creencias epistemológicas sobre la enseñanza y aprendizaje}

Diferentes investigaciones muestran que las creencias epistemológicas que se poseen sobre las matemáticas tienen relación de manera explícita o implícita con la práctica, y por tanto con las que tiene el docente en ejercicio o en formación frente a los procesos de enseñanza y aprendizaje de las matemáticas (Penn, 2012; Steiner, 1987, White-Fredette, 2009/2010). Para este estudio se toman como referentes posturas de tipo tradicional y constructivista que desde la literatura se considera que están relacionadas con posturas de tipo absolutista y falibilista, respectivamente.

En la visión tradicional del aprendizaje de las matemáticas se piensa que el profesor es quien posee e imparte conocimiento, por tanto es el centro del proceso, en tanto que el estudiante es un receptor pasivo del conocimiento, recibe información de su profesor o libros, tiene el papel de asimilar, mecanizar algoritmos, memorizar y usar conceptos, generalmente en situaciones de tipo rutinario y repetitivo, donde las respuestas son correctas o incorrectas. Se considera que el estudiante logra aprender si es capaz de repetir la información que provee el maestro. En coherencia con esto, la enseñanza en este paradigma se aborda como un proceso de transmisión de conocimientos, generalmente de manera magistral por parte del docente, mientras que el estudiante escucha, mecaniza y practica. El docente conduce a los estudiantes a lograr la respuesta correcta, a que puedan reproducir información o procedimientos luego de ser presentados. En este enfoque se llega a una forma extrema de enseñanza, descrita como un modelo autoritario de transmisión que ocurre cuando el docente impone a los estudiantes los métodos que deben usar para resolver problemas, es decir, impone restricciones (Ernest, 1991).

En contraste, desde el punto de vista constructivista del aprendizaje se considera que el conocimiento lo construyen activamente las personas a partir de sus conocimientos previos, por lo cual se deben crear entornos de aprendizaje que permitan el descubrimiento, el desarrollo del pensamiento crítico y el trabajo en equipo; los estudiantes deben ser investigadores activos y el docente facilita el aprendizaje planteando preguntas desafiantes (Conner, Edenfield, Gleason y Ersoz, 2011). El lenguaje influye y es importante en la construcción del conocimiento por parte de las personas, ya que a través de éste se justifica y comunica (Ernest, 1991; Phillips, 2000). Se considera que el estudiante logra el aprendizaje cuando puede resolver problemas, tiempo después del período de instrucción y en una amplia variedad de situaciones. Frente a la enseñanza, según el 
enfoque constructivista no hay una manera única, se deben proveer al estudiante escenarios que le permitan construir conocimientos matemáticos, es preciso motivarlo para que explore ideas, haga conjeturas y luego las justifique, de modo que a través del razonamiento matemático pueda argumentar sus afirmaciones y convencer a sus compañeros y maestros. También es importante el trabajo en solución de problemas y que se incluyan aplicaciones del mundo real (Ball y Bass, Penn, 2012; Steiner, 1987, White-Fredette, 2009/2010).

\section{Metodología}

Se utilizó un enfoque cualitativo y una metodología de estudio de casos que permitiera profundizar sobre el fenómeno investigado. El estudio se realizó en dos fases: en la primera, participaron seis docentes en formación, tres hombres y tres mujeres, de la Licenciatura en Matemáticas de la Universidad Antonio Nariño, Colombia, quienes durante el primer semestre de 2015 estaban cursando séptimo u octavo semestre. En la segunda fase participaron tres docentes en ejercicio, dos hombres y una mujer. A todos los participantes se les informó el objetivo del estudio, se les invitó a participar de manera voluntaria y ellos firmaron un consentimiento informado. Los docentes en formación se identifican en este estudio con los nombres de Enrique, Yeny, Edwin, Jairo, Lucía y Yadira; los docentes en ejercicio, como John, Myriam y Francisco.

Para el desarrollo de la investigación se realizaron dos cursos, uno dirigido a docentes en formación y otro a docentes en ejercicio. Ambos fueron diseñados con énfasis en la epistemología, la filosofía y la historia de las matemáticas, y tuvieron un componente de trabajo en solución de problemas a través de conjeturas. El curso de docentes en ejercicio tuvo como objeto de estudio una de las partes finales del curso de docentes en formación.

Los seis docentes en formación participaron de un curso sobre epistemología y filosofía de las matemáticas, que se desarrolló durante dieciséis semanas, en el primer semestre de 2015. El objetivo del curso fue estudiar y conocer diferentes corrientes filosóficas y posturas epistemológicas sobre las matemáticas y su enseñanza, de manera que los participantes pudieran construir, reconstruir o modificar completamente la propia.

Por su parte, los docentes en ejercicio participaron en un curso que tuvo como eje un recorrido histórico a través del estudio de la solución de ecuaciones cuadráticas en una variable, el cual se desarrolló entre septiembre y noviembre de 2015, con cinco encuentros presenciales en total, cada uno de tres horas. Se buscaba, a través del recorrido histórico propuesto en el tema concreto de las ecuaciones cuadráticas, analizar con los docentes en ejercicio los problemas epistemológicos y científicos que

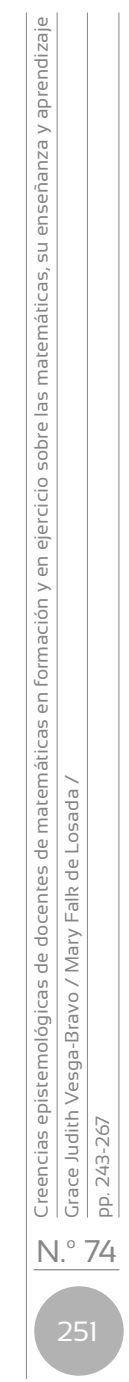


tuvieron que enfrentar los matemáticos en las diferentes épocas en la búsqueda de soluciones de ecuaciones cuadráticas, y analizar la importancia de la historia de las matemáticas para su desarrollo y para el proceso de enseñanza-aprendizaje. Mediante este recorrido, se buscaba además que los docentes en ejercicio reflexionaran acerca de las creencias que tienen sobre las matemáticas, su enseñanza y aprendizaje.

Aunque los dos cursos no fueron exactamente iguales, sí tenían como propósito principal servir como escenarios de aprendizaje en los cuales los participantes reflexionaran sobre sus creencias epistemológicas acerca de las matemáticas, su enseñanza y aprendizaje, así como identificar los aspectos más relevantes que permiten dicha reflexión.

Se diseñaron dos cuestionarios cerrados. El primero, se denominó "Creencias epistemológicas acerca de las matemáticas", con un total de 29 afirmaciones para identificar posturas de tipo absolutista o falibilista sobre tales creencias. El segundo, Ilamado "Creencias acerca de la enseñanza y aprendizaje de las matemáticas", contiene un total de 20 afirmaciones, para identificar posturas tradicionales o constructivistas sobre la enseñanza y aprendizaje de este saber. Los cuestionarios se diseñaron para este estudio con base en otros instrumentos utilizados en diferentes investigaciones relacionadas con las creencias acerca de la naturaleza de las matemáticas y su enseñanza (Penn, 2012; Walker, 2007) y se validaron mediante un procedimiento de juicio de expertos (véanse los anexos 1 y 2). Para cada afirmación los participantes debían marcar en una escala Likert qué tanto se identificaban o estaban de acuerdo. Se utilizó una graduación de 1 a 5, dónde 1 significaba completamente en desacuerdo, 2 en desacuerdo, 3 ni en desacuerdo ni de acuerdo, 4 de acuerdo y 5 completamente de acuerdo. Se aclaró y escribió en cada instrumento que no había afirmaciones correctas o incorrectas.

Los participantes respondieron los cuestionarios al comienzo y al final del curso. Con esta información se determinó, en cada momento, la postura epistemológica reportada por cada participante o la tendencia que podía observarse. Para esto se hizo un análisis del número de afirmaciones de cada tendencia falibilista o absolutista, y constructivista o tradicional con las que cada participante se identificó. Teniendo en cuenta la escala de graduación de los instrumentos se consideró que había un cambio significativo de creencia entre una y otra aplicación si el valor absoluto de la diferencia entre los valores marcados era igual o mayor a dos. Con base en las respuestas dadas en los instrumentos cerrados a cada participante se realizaron entrevistas semiestructuradas con el fin de indagar y profundizar sobre la justificación y el origen de las creencias señaladas, y específicamente en la segunda entrevista para identificar los cambios presentados, su justificación y percepción del curso realizado. También se incluyeron preguntas relacionadas con el impacto de su formación de pregrado en la construcción de sus creencias. 


\section{Resultados}

A continuación se presentan los resultados, enmarcados en categorías relacionadas con las preguntas de investigación.

\section{Creencias epistemológicas sobre las matemáticas, su enseñanza y aprendizaje al inicio y final de cada curso}

En este estudio se pudieron evidenciar diferencias y similitudes importantes en cada uno de los dos grupos (profesores en formación y profesores en ejercicio). En la figura 1 se muestra la postura identificada, a partir de los instrumentos, para cada participante al inicio y al final de cada curso. Se ejemplifica con la magnitud y dirección del vector, la posición inicial y final, y los cambios reportados.

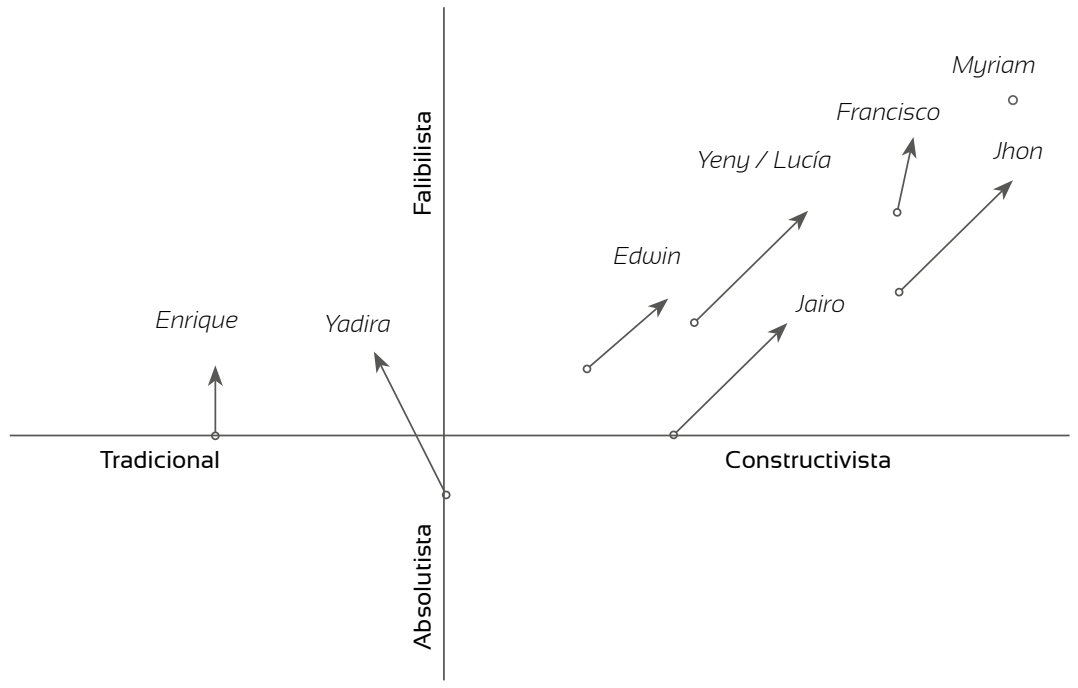

Figura 1. Creencias epistemológicas de docentes en formación y en ejercicio sobre las matemáticas, su enseñanza y aprendizaje, reportadas al comienzo y al final de cada curso

Fuente: elaboración propia

En primer lugar, a partir de los instrumentos cerrados, se evidenció que los docentes en ejercicio reportan posturas definidas y coherentes entre sí. En contraste, las reportadas por los docentes en formación son más tentativas, pues los cambios en las respuestas dadas a los puntos de los instrumentos revelan que se encuentran en proceso de construcción o consolidación.

Los tres docentes en ejercicio se identificaron, desde el comienzo, con una postura falibilista acerca de las matemáticas y constructivista frente a su enseñanza y aprendizaje, la cual mantuvieron hasta el final, 
aunque señalaron algunos cambios, lo que permiten ver que están abiertos a la reflexión. En el caso de los docentes en formación, al inicio no se pudo identificar una postura clara de Enrique ni de Jairo acerca de sus creencias epistemológicas, y Yadira cambió de absolutista a falibilista. Con respecto a la enseñanza y aprendizaje de las matemáticas, Yadira no se identificó con ninguna tendencia al comienzo del curso. Los demás estudiantes, aunque se identificaron desde el inicio con posturas falibilista y constructivista, que fueron consolidando, también señalaron, especialmente en la primera aplicación de los instrumentos, algunas posturas contrarias. Se observó durante las entrevistas que los docentes en formación tenían dificultad para argumentar las creencias señaladas, por lo que las cambiaban, aparentemente para no dar explicaciones; esa fue la constante en el caso de Enrique. Los docentes en ejercicio siempre se mostraron seguros durante las entrevistas, y aunque algunas creencias podían ser contrarias a la postura identificada, argumentaban sobre ella con mucha propiedad.

\section{Estructuración de las creencias}

Sobre la estructuración de las creencias, los docentes en ejercicio expresan con claridad que su formación formalista en el pregrado y su experiencia docente han sido los aspectos que les han aportado. Para los tres fue positivo e importante el haber recibido en el pregrado una formación matemática rigurosa, de corte formalista, a través de las asignaturas disciplinares, esto es, las que orientaban los matemáticos puros. Pero, al mismo tiempo, señalan las dificultades que han tenido que superar para su ejercicio docente, pues al comienzo las creencias formadas sobre las matemáticas en el pregrado, orientadas al formalismo, eran un obstáculo, en lugar de un apoyo, para su trabajo de aula. Esto lo señaló especialmente John, quien afirmó que al iniciar su proceso de práctica quiso hacerlo al estilo formalista y se dio cuenta que no era pertinente, y fueron los docentes de corte pedagógico y didáctico quienes lo orientaron. Este hallazgo ratifica lo señalado por Sfard, citada por White-Fredette (2009/2010), acerca de que los matemáticos puros forman parte del paradigma absolutista, los investigadores en educación matemática del falibilista, y los docentes de matemáticas de educación básica y media están atrapados en el medio, con una mayor tendencia al formalismo. Se puede inferir de lo expresado en las entrevistas, que por su fuerte formación formalista en el pregrado, los docentes en ejercicio inicialmente tenían creencias orientadas a esa postura, pero que, sobre todo su experiencia docente, y de alguna manera por participar continuamente en diferentes eventos de matemática y educación matemática, han ido transformándola. 
Por otra parte, los docentes en ejercicio establecen una diferencia entre la matemática como disciplina científica y la matemática escolar. A pesar de las dificultades señaladas, siguen considerando que es fundamental que se forme a los futuros docentes con algo de rigor matemático, aunque señalan que deben existir otros espacios de formación que ayuden a articular las matemáticas con su proceso de enseñanza y aprendizaje.

Por su parte, los docentes en formación -quienes reportan menos consolidadas sus creencias epistemológicas acerca de las matemáticas, su enseñanza y aprendizaje- inicialmente hicieron más referencia a su formación en el colegio, a la forma de enseñar de sus docentes y también a su experiencia docente; aludieron muy poco a su formación universitaria. Para Lucía y Yadira, el programa les ha enseñado que es importante la demostración cuidadosa de las afirmaciones y dijeron que han aprendido más matemáticas.

\section{Posible impacto de los cursos}

Se pudo evidenciar que el incorporar experiencias de aprendizaje basadas en la filosofía, la epistemología y la historia de las matemáticas, en las cuales se generan espacios implícitos, pero especialmente explícitos para que los docentes en formación y en ejercicio reflexionen sobre sus creencias epistemológicas acerca de las matemáticas, su enseñanza y aprendizaje, ayuda a la formación, transformación o consolidación de las mismas (Charalambous, Panaoura y Philippou, 2009; Flores, 1995; White-Fredette, 2009).

Se observó que todos los estudiantes en formación tuvieron cambios importantes, el curso les ayudó en la consolidación de sus creencias, las cuales siguen en proceso de consolidación. Y aunque los docentes en ejercicio tenían aparentemente más consolidadas sus creencias, también están abiertos a ampliarlas y a incorporar nuevos elementos. Todos los docentes participantes hicieron referencia al impacto generado por el estudio de la obra de Lakatos y los aportes de Davis y Hersh, especialmente con su reflexión acerca del anverso y el reverso de las matemáticas.

Por ejemplo, Enrique, que se identifica con una postura tradicional frente a la enseñanza y aprendizaje de las matemáticas, dijo en la entrevista final que le gustaría hacer cosas nuevas con sus estudiantes, que ellos sean más protagonistas de su aprendizaje, que participen y trabajen por cuenta propia, pero señala una presión por cumplir con los contenidos establecidos y que eso no se lo permite. También muestra su preocupación por seguir enseñando de manera tradicional, diciendo que debe trabajar al respecto y que en su tesis de grado, que iniciará el siguiente semestre, lo va a intentar. 
Jairo dice que durante el curso tuvo sus propias crisis, considera que fue innovador, que se logró articular lo teórico con lo práctico y lo disciplinar con lo pedagógico. Señaló que generalmente las matemáticas van por una parte y la pedagogía por otra. Y Yadira, aunque también señaló una postura tradicional frente a la enseñanza y aprendizaje de las matemáticas, dijo que el curso le permitió reflexionar sobre cómo está enseñando, si lo que hace en el aula es más de tipo formal o si, por el contrario, lo hace de manera más cuidadosa y se tiene en cuenta el anverso y reverso de las matemáticas, y la historia de las matemáticas.

Otro aspecto que evidencia el impacto del curso en los docentes en formación, especialmente para aportar en la continua reflexión, es que varios hablaron del dilema ético que debían enfrentar, al ser conscientes de la importancia de permitir a los estudiantes explorar, razonar, crear y descubrir por cuenta propia, pero al mismo tiempo lo que eso implica en dedicación y esfuerzo de su parte en la preparación de sus clases. Sobre esto hablaron Enrique, Yeny, Jairo y Lucía.

De los docentes en ejercicio, John avanzó de manera más significativa en la consolidación de sus creencias. Señaló, como lo hicieron los docentes en formación, la importancia de permitir a los estudiantes llegar al conocimiento matemático por su propia cuenta en lugar de presentarlo ya terminado, para que simplemente sea memorizado y usado de manera mecánica. John también señaló que la presión por cumplir con el currículo establecido no le permite trabajar de manera más significativa la matemática, pues debe además ceñirse a lo establecido por el libro de texto. Esta presión también la señaló Enrique.

Lo descrito permite afirmar que la experiencia docente intensiva, esto es, de muchos años, es importante para la formación y consolidación de creencias, como se evidenció con los docentes en ejercicio; en cambio, la formación recibida en el pregrado es de difícil y lenta asimilación, como se aprecia con los docentes en formación, quienes no señalan un impacto significativo de su programa de formación, en contraste con lo reportado por los docentes en ejercicio. Por otra parte, los hallazgos muestran que algunos docentes, tanto en formación como en ejercicio, han construido un sistema de atenuantes, por ejemplo el currículo y los textos que se les han impuesto, para justificar la contradicción entre lo que reportan son sus creencias frente a la enseñanza y aprendizaje de las matemáticas y lo que suelen hacer en el aula, y también parecen estar cómodos con relación a esa situación. 


\section{Consideraciones para los programas de formación}

A partir de los hallazgos de esta investigación y la revisión de la literatura al respecto se pueden señalar algunos aspectos que deben tenerse en cuenta en los programas de formación inicial, de manera que puedan contribuir a que los futuros docentes construyan o consoliden creencias epistemológicas sobre las matemáticas, su enseñanza y aprendizaje coherentes con los fines de la educación matemática. Estas recomendaciones se extienden a programas de formación posgradual o permanente, ya que como señalan Charalambous, Panaoura y Philippou (2009) es posible que los programas de formación inicial sean capaces de aportar en la construcción o consolidación de creencias, pero otros factores como el entorno escolar y las comunidades a las que pertenecen los docentes son importantes para el éxito sostenido de cualquier esfuerzo.

En primer lugar, en esta investigación se pudo observar que, en las creencias de los docentes por un lado hay una disciplina formal que se debe aprender de manera rigurosa, donde la demostración cuidadosa es un requisito, esto es, una tendencia de tipo más formalista. Por otro lado, en el momento de ir al aula, escenario en el cual la matemática misma parece inútil, se deben utilizar herramientas distintas. En este sentido, el contraste epistemológico entre la perspectiva que se da a las matemáticas, como sistema axiomático formal, y la que se requiere, tanto de las matemáticas como de la educación matemática, para que se enriquezcan mutuamente, emerge con claridad en las creencias de los sujetos participantes en esta investigación.

El enfoque epistemológico formal y axiomático de las matemáticas que se ofrece en los programas de formación de futuros profesores, como lo expresaron especialmente los docentes en ejercicio, la divorcia por completo de la matemática escolar, a pesar de que ésta claramente corresponde a partes seleccionadas de la matemática elemental. En consecuencia, esa formación se torna prácticamente irrelevante e impide que informe la práctica de los futuros profesores y contribuya al éxito de reformas curriculares que buscan transformarla.

En este sentido, una recomendación que emerge es la incorporación durante la formación inicial y continua, de manera explícita y permanente, de experiencias que permitan a los futuros docentes o a docentes en ejercicio confrontar sus creencias y sus implicaciones en su práctica docente y reflexionar sobre ellas, como lo señalan otros estudios (Cooney, Shealy y Arvold, 1998; Cross, 2009; Hersh, 1997).

El trabajo de campo realizado mostró que todos los participantes, tanto docentes en formación como en ejercicio, se sintieron impactados y señalaron cambios en algunas de sus creencias, por aspectos considerados en los cursos, especialmente de orden histórico, epistemológico y filosófico. 
Todos los docentes en ejercicio, y al menos la mitad de los docentes en formación, se refirieron explícitamente a la importancia de estudiar y profundizar en la historia de las matemáticas, y su aporte para mejorar los procesos de enseñanza y aprendizaje de este saber. En el caso de los docentes en formación, destacaron el poder profundizar en un tema concreto y ver todo su desarrollo histórico, indicando que durante su formación nunca tuvieron un ejercicio parecido.

Yeny, por ejemplo, señaló que el recorrido histórico sobre la epistemología de las matemáticas en las escuelas medieval y griega le permitió conocer las problemáticas que enfrentaron los matemáticos de la época alrededor del conocimiento matemático y su interés de hacer de las matemáticas algo universal, lo cual considera fundamental en su ejercicio docente. Myriam destacó como un aporte del curso el haber abordado la historia de las matemáticas alrededor de un tema específico, lo cual le permite reflexionar sobre la importancia de ir a la historia cuando se van a abordar los diferentes temas. Y Francisco incluso hizo alusión a la importancia de la historia en la formación de futuros docentes, argumentando que es muy útil para el trabajo en el aula y permite no solo construir conocimiento con las estudiantes, sino también formar valores, por ejemplo al ver la pasión y el empeño de muchos matemáticos por comprender, conocer, descubrir y aprender.

Como lo señalan Fauvel y Van Maanen (2000), la historia de las matemáticas aporta, entre otros aspectos, comprensión profunda tanto de las matemáticas como de otros temas, permite ver su evolución y comprender que las matemáticas son el resultado de una comunidad, aporta conocimiento sobre dificultades presentadas en diferentes momentos y esto a su vez ayuda a que los estudiantes adquieran confianza. A través del estudio de la historia de las matemáticas se incorporan además aspectos filosóficos y epistemológicos que también deben tenerse en cuenta durante la formación docente (Chassapis 2007; White-Fredette, 2009/2010).

Por otra parte, todos los docentes participantes hicieron referencia a la importancia y el impacto generado por el estudio y la reflexión alrededor de aspectos de orden filosófico y epistemológico. Señalaron como novedoso y significativo el estudio de las posturas de Lakatos, Davis y Hersh, afirmando que los hizo reflexionar sobre la manera en que desarrollan su práctica docente, preguntarse qué es la matemática, qué se conoce acerca de la creación matemática, y sobre la distancia que generalmente existe entre la forma como la matemática ha sido creada y lo que se presenta en el aula, Ilamado por Hersh el anverso y el reverso de las matemáticas. 
Por ejemplo Enrique, quien no tenía una postura clara al comienzo sobre las matemáticas y al finalizar avanzó hacia una de tipo falibilista -manteniendo, sin embargo, una postura tradicional frente a su enseñanza y aprendizaje-, señaló al terminar el curso que, a partir de lo estudiado de Lakatos, es consciente de que debe permitir que los estudiantes sean más protagonistas de su aprendizaje, que participen y trabajen por cuenta propia.

En articulación con estos hallazgos, en los estándares de competencias de matemáticas en Colombia se afirma que para que los docentes puedan desarrollar competencias matemáticas en sus estudiantes se requiere que adopten un modelo epistemológicamente coherente sobre las propias matemáticas. Para ello, también señalan los estándares, que con base en nuevas tendencias de la filosofía de las matemáticas, es necesario que los docentes reflexionen, exploren y se apropien de supuestos sobre las matemáticas, como que son

\begin{abstract}
... una actividad humana inserta en y condicionada por la cultura y por su historia en la cual se utilizan distintos recursos lingüísticos y expresivos para plantear y solucionar problemas [...]. En la búsqueda de soluciones y respuestas a estos problemas surgen progresivamente técnicas, reglas y sus respectivas justificaciones, las cuales son socialmente decantadas y compartidas, y que son también el resultado acumulado y sucesivamente reorganizado de la actividad de comunidades profesionales. (MEN, 2002, p. 49).
\end{abstract}

Asimismo, varias investigaciones señalan como componentes esenciales en la formación profesional de docentes de matemáticas la filosofía y la epistemología de las matemáticas. Las creencias que tienen los docentes sobre este saber, su enseñanza y aprendizaje reflejan, generalmente de manera implícita, una filosofía de las matemáticas, que se constituye en una especie de práctica filosófica y muchas veces prevalece sobre los conocimientos matemáticos (Chassapis, 2007; White-Fredette; 2009/2010).

El estudio de la filosofía de las matemáticas debe servir a los docentes para ser más reflexivos sobre su práctica, debe darles elementos para la definición de propósitos y objetivos de su trabajo, y permitirles una comprensión más profunda de las matemáticas como su objeto de conocimiento. El estudio de la filosofía de las matemáticas como parte de la formación docente, señala White-Fredette (2009/2010), ayuda a los docentes a integrar la enseñanza desde el enfoque constructivista más fácilmente mediante el estudio de nuevas tendencias orientadas hacia una epistemología de las matemáticas de corte falibilista.

Los elementos señalados permiten concluir que lo deseable para los programas de formación es construir una propuesta curricular en la cual se articulen la historia, la filosofía y la epistemología de las matemáticas como hilos conductores para el estudio de las matemáticas y, a través de 
ese conocimiento profundo, se consolide un trabajo intensivo en solución de problemas que sea el puente entre la matemática como disciplina científica y la matemática escolar, como subconjunto que es de la matemática elemental. Sobre este aspecto se requiere más investigación.

\section{Conclusiones}

Este estudio permitió conocer las creencias epistemológicas de docentes de matemáticas en formación y en ejercicio desde la perspectiva de los participantes, por tanto vale la pena señalar que esto no da lugar a generalizaciones y se requiere más investigación en este campo. Se pudieron identificar aspectos sobre la manera como se han ido estructurando dichas creencias, así como los cambios que pueden tener cuando los docentes participan en diferentes cursos de formación, que buscaban de manera explícita desafiar sus creencias.

Los cursos diseñados y realizados para esta investigación generaron en los nueve participantes inquietudes que les permitieron reflexionar, de manera clara, sobre sus creencias y su impacto o posible impacto en sus prácticas. Los cursos incorporaron explícitamente aspectos epistemológicos, filosóficos e históricos, así como un componente para el trabajo en solución de problemas. Estos aspectos son señalados por diferentes investigaciones como relevantes para la construcción y consolidación de creencias, como se describió.

Los resultados encontrados permitieron hacer algunas recomendaciones para los programas de formación inicial y continua, de modo que puedan incorporar cambios en sus diseños curriculares encaminados a que los docentes en formación o en ejercicio consoliden creencias de acuerdo con los fines de la educación matemática. Se espera que de ese modo las reformas curriculares que se vienen proponiendo desde comienzos del siglo XXI, enfocadas al desarrollo de competencias en los estudiantes, puedan tener mayor éxito del que hasta ahora se ha evidenciado a través de las diferentes pruebas nacionales e internacionales que presentan los estudiantes colombianos (ICFES, 2010, 2013, 2016).

\section{Recomendaciones}

Dado el carácter de este estudio, en el cual participaron seis docentes en formación y tres docentes en ejercicio, no se pueden generalizar los hallazgos. No obstante, sí provee información importante y se espera que dé lugar a nuevos estudios que permitan tener una mayor y mejor comprensión sobre el sistema de creencias y su impacto en la práctica. 
En primer lugar, teniendo en cuenta lo complejo que es el sistema de creencias, es necesario desarrollar nuevos diseños metodológicos que permitan informar con mayor precisión las creencias epistemológicas sobre las matemáticas, su enseñanza y aprendizaje que tienen docentes en formación, en ejercicio y formadores, y la coherencia entre éstas. Otros estudios pueden estar orientados a definir nuevas y más precisas categorías sobre las creencias de docentes en formación y en ejercicio, de acuerdo con las tendencias actuales y de vanguardia en educación matemática y el impacto que éstas generan a su vez en el desarrollo de competencias matemáticas de sus estudiantes.

Es recomendable hacer estudios longitudinales que reporten las creencias que tienen docentes en formación cuando empiezan su programa, cómo se van transformando o consolidando a medida que avanzan en el mismo, el impacto que puede tener el proceso de práctica docente, y contrastar con las creencias que reportan al finalizar el programa. Este tipo de estudios puede dar información importante para analizar la pertinencia lograda por los programas académicos.

Por otra parte, y teniendo en cuenta que uno de los hallazgos de este estudio hace referencia a la necesidad de hacer de la historia, la filosofía y la epistemología de las matemáticas hilos conductores en los programas de formación, se requiere investigación sobre maneras de hacerlo, qué tipo de nuevos materiales y metodologías es necesario diseñar e implementar y ver el impacto que pueden tener. El conocimiento profundo y continuo de la historia, la filosofía y la epistemología de las matemáticas debe permitir a los programas de formación y a sus egresados ir más allá; y en coherencia con la construcción de creencias más productivas hacia las matemáticas y su aprendizaje, debe servir para cuestionar lo que se ha establecido debe ser la educación matemática de niños y jóvenes, sus fines, argumentar si es lo pertinente o no, y en su defecto liderar el diseño de currículos más retadores.

En articulación con esto, se requiere investigación que aborde las preocupaciones señaladas tanto por docentes en formación como en ejercicio sobre la presión que sienten por cumplir con un listado de temas preestablecidos, aspecto que señalan como una causa para no incorporar nuevas tendencias en el proceso de aprendizaje de las matemáticas. Se requieren estudios que muestren también cuáles son las creencias que tienen los formadores de formadores y que se indague sobre las creencias que se espera en los programas de formación que los estudiantes construyan o consoliden, y las estrategias establecidas en su diseño curricular para lograrlo. 


\section{Referencias}

Agudelo-Valderrama, C. (2006). The growing gap between colombian education policy, official claims and classroom realities: Insights from mathematics teachers' conceptions of beginning algebra and its teaching purpose. International Journal of Science and Mathematics Education, 4, 513-544.

Artz, A. y Armour-Thomas, E. (1999). A cognitive model for examining teachers' instructional practice in mathematics: A guide for facilitating teacher reflection. Educational Studies in Mathematics, 40, 211-235.

Charalambous, C.; Panaoura, A. y Philippou, G. (2009). Using the history of mathematics to induce changes in preservice teachers' beliefs and attitudes: insights from evaluating a teacher education program. Educational Studies in Mathematics, 71, 161-180. DOI: 10.1007/ s10649-008-9170-0

Chassapis, D. (2007). Integrating the philosophy of mathematics in teacher training courses. En Philosophical dimensions in mathematics education (pp. 61-79). Springer US.

Colombia, Ministerio de Educación Nacional. (1998). Matemáticas. Lineamientos curriculares. Bogotá: autor.

Colombia, Ministerio de Educación Nacional. (2006). Estándares básicos de competencias en Lenguaje, Matemáticas, Ciencias y Ciudadanas. Guía sobre lo que los estudiantes deben saber y saber hacer con lo que aprenden. Bogotá: autor.

Conner, A.; Edenfield, K.; Gleason, B. y Ersoz, F. (2011). Impact of a content and methods course sequence on prospective secondary mathematics teachers' beliefs. Journal of Mathematics Teacher Education, 14, 483504. DOI:10.1007/s10857-011-9186-8

Cooney, T.; Shealy, B. y Arvold, B. (1998). Conceptualizing belief structures of preservice secondary mathematics teachers. Journal for Research in Mathematics Education, 29(3), 306-333.

Cross, D. (2009). Alignment, cohesion, and change: Examining mathematics teachers' belief structures and their influence on instructional practices. Journal of Mathematics Teacher Education, 12, 325-346. DOI 10.1007/s10857-009-9120-5.

Cross, D. (2015). Dispelling the notion of inconsistencies in teachers' mathematics beliefs and practices: A 3-year case study. Journal of Mathematics Teacher Education, 18, 173-201. DOI 10.1007/s10857014-9276-5.

Davis, P.; Hersh, R. y Marchisotto, E. (2012). The mathematical experience. Boston: Birkhäuser. 
Ernest, P. (1989). The impact of beliefs on the teaching of mathematics.

En C. Keitel, P. Damerow, A. Bishop y P. Gerdes (eds.). Mathematics, education, and society (pp. 99-101). París:UNESCO.

Ernest, P. (1991). Philosophy of mathematics education. Nueva York: Falmer.

Ernest, P. (1998). Social constructivism as a philosophy of mathematics. Albany, Ny: State University of New York Press.

Fauvel, J. y Van Maanen, J. (eds.): (2000). History in mathematics educationThe ICMI study. Dordrecht: Kluwer.

Flores, P. (1995). Concepciones y creencias de los futuros profesores sobre las matemáticas, su enseñanza y aprendizaje. Evolución durante las prácticas de enseñanza (tesis doctoral). Universidad de Granada, Granada.

Handal, B. y Herrington, A. (2003). Mathematics teachers' beliefs and curriculum reform. Mathematics Education Research Journal, 15(1), 59-69.

Hersh, R. (1997). What is mathematics really? Nueva York: Oxford University Press.

Instituto Colombiano para la Evaluación de la Educación (ICFES). (2010). Resultados de Colombia en timss 2007. Resumen ejecutivo. Bogotá: autor.

Instituto Colombiano para la Evaluación de la Educación (ICFES). (2013). Colombia en PISA 2012. Informe nacional de resultados. Resumen ejecutivo. Bogotá: autor.

Instituto Colombiano para la Evaluación de la Educación (ICFES). (2016). Colombia en PISA 2015. Resumen ejecutivo. Bogotá: autor.

Lakatos, I. (1976). A renaissance of empiricism in the recent philosophy of mathematics. The British Journal for the Philosophy of Science, 27(3), 201-223.

Lerman, S. (1990). Alternative perspective of the nature of mathematics. British Educational Research Journal, 16, 53-61.

Organización de las Naciones Unidas para la Educación, la Ciencia y la Cultura (Unesco). (2014). Enseñanza y aprendizaje: Lograr la calidad para todos. París: EdicionesunesCo.

Pajares, M. F. (1992). Teachers' beliefs and educational research: Cleaning up a messy construct. Review of Educational Research, 62(3), 307-333.

Pantziara, M.; Karamanou, M. y Philippou, G. (2013). Teachers'beliefs and knowledge related to the Cyprus mathematics curriculum reform. En F. Arzarello (Presidencia), Proceedings of the Eighth Congress of European Research in Mathematics Education (Cerme 8). Manavgat-Side, AntalyaTurquía.

Penn, A. (2012). The alignment of preservice elementary school teachers' beliefs concerning mathematics and mathematics teaching (tesis de maestría). Queen's University, Kingston, Ontario, Canada. 
Pepin, B. (1999). Epistemologies, beliefs and conceptions of mathematics teaching and learning: The theory, and what is manifested in mathematics teachers' work in England, France and Germany. TNTEE Publications, 2(1), 127-146.

Phillips, D. C. (2000). An opinionated account of the constructivist landscape. En D. C. Phillips (eds.). Constructivism in Education, (pp. 1-18). Chicago, Illinois: The University of Chicago Press.

Putnam, H. (1975). Mathematics, matter and method. Philosophical Papers (volume 1). Cambridge: Cambridge University Press.

Raymond, A. (1997). Inconsistencies between a beginning elementary teacher's mathematics beliefs and teaching practice. Journal for Research in Mathematics Education, 28(5), 550-576.

Roscoe, M. y Sriraman, B. (2011). A quantitative study of the effects of informal mathematics activities on the beliefs of preservice elementary school teachers. Zdm, 43(4), 601. DOI:10.1007/s11858-011-0332-7.

Steiner, H. (1987). Philosophical and epistemological aspects of mathematics and their interaction with theory and practice in mathematics education. Learning of Mathematics 7(1), 7-13.

Thompson, A. (1984). The relationship of teachers' conceptions of mathematics teaching to instructional practice. Educational Studies in Mathematics, 15, 105-127.

Thompson, A. (1992). Teacher's beliefs and conceptions: A synthesis of the research. En D. A. Grouws (ed.). Handbook on mathematics teaching and learning (pp. 127-146). Nueva York: Macmillan.

Valero, P. (2017). El deseo de acceso y equidad en la educación matemática. Revista Colombiana de Educación, (73), 99-128

Walker. (2007). The development and construct validation of epistemological beliefs survey for mathemathics (tesis doctoral). Oklahoma State University, EEUU.

White-Fredette, K. (2009). What is mathematics? An exploration of teachers' philosophies of mathematics during a time of curriculum reform. Middlesecondary education and instructional technology dissertations. Paper 46.

White-Fredette, K. (2009/2010). Why not philosophy? Problematizing the philosophy of mathematics in a time of curriculum reform. The Mathematics Educator, 19(2), 21-31.

Yang, X. (2014). Conception and characteristics of expert mathematics teachers in China. Berlin: Springer. 


\section{Anexo 1. Cuestionario}

\section{Creencias epistemológicas acerca de la matemática}

1. La matemática es una creación de la mente humana.

2. La matemática está por ahí, en el universo, esperando a ser descubierta.

3. La matemática se construye a partir de la experiencia humana.

4. La matemática consiste, en su mayoría, en hechos y procedimientos que se tienen que aprender y/o ser aceptados como verdaderos.

5. Cualquier persona puede crear o descubrir hechos matemáticos por su propia cuenta.

6. Solo los matemáticos pueden hacer nueva matemática.

7. En matemática algo es verdadero solo si se demuestra rigurosamente por medio del uso de la lógica y el razonamiento.

8. Las teorías matemáticas son en gran parte producto de la creatividad.

9. Los problemas son menos importantes que los teoremas.

10. La matemática es una ciencia formal y exacta, no hay lugar para la conjetura.

11. Cada día se inventa nueva y mucha matemática.

12. El conocimiento matemático es falible y corregible, como cualquier ciencia humana.

13. El conocimiento matemático es cierto, objetivo e incuestionable.

14. En matemáticas, las respuestas son correctas o incorrectas.

15. Los procedimientos y reglas matemáticas no cambian.

16. Los resultados de los problemas de matemáticas son siempre predecibles.

17. Es posible inventar problemas matemáticos que no tienen solución.

18. La matemática está en continua evolución.

19. La matemática ha evolucionado a través de la historia.

20. La mayor parte de lo que es verdad en las matemáticas ya se conoce.

21. Acerca de toda la matemática actual no se puede tener total certeza.

22. En matemáticas las respuestas a las preguntas pueden cambiar a medida que se tiene más información.

23. Puede haber muchas formas diferentes de resolver un problema matemático.

24. Hacer matemáticas es una actividad solitaria. 
25. La matemática es una ciencia formal y exacta, no hay lugar para la contradicción.

26. Las matemáticas son un conjunto de reglas, fórmulas, hechos y procedimientos.

27. El conocimiento matemático es absolutamente cierto, incuestionable y objetivo.

28. Hacer matemáticas es una actividad que genera nuevo conocimiento.

29. Para entender las matemáticas es importante relacionarlas con la vida real.

\section{Anexo 2. Cuestionario}

\section{Creencias acerca de la enseñanza de las matemáticas}

1. Cualquier persona puede aprender matemáticas.

2. Para aprender matemáticas se requiere de habilidades especiales hacia la matemática.

3. Los estudiantes pueden ser creativos y descubrir hechos matemáticos por su propia cuenta.

4. El docente de matemáticas es el responsable de transmitir el conocimiento matemático a sus estudiantes.

5. Los estudiantes pueden resolver problemas de manera creativa aun cuando no tengan muchos conocimientos matemáticos.

6. El éxito del aprendizaje de las matemáticas está en la repetición de procedimientos.

7. En el aprendizaje de las matemáticas es fundamental la memorización de conceptos.

8. Los errores en la clase de matemáticas son importantes y una fuente de nuevo aprendizaje, por lo cual se deben discutir en clase.

9. Los errores de los estudiantes se deben discutir en la clase como ejemplo de lo que no se debe hacer.

10. Los temas de la matemática escolar están claramente establecidos y son estables en el tiempo.

11.Lo que es más importante en la solución de un problema es la respuesta, no las ideas que pueda tener el estudiante sobre cómo encontrarla.

12. En la clase de matemáticas es importante que se muestre a los estudiantes problemas sin solución así como diferentes formas de ver y resolver un mismo problema. 
13. Los problemas matemáticos deben tener una respuesta exacta para que el estudiante pueda saber si está trabajando correctamente.

14. La clase es una comunidad de aprendizaje donde docentes y estudiantes interactúan para construir y validar conocimiento matemático.

15. Los estudiantes deben aprender y reconocer que la matemática es una ciencia formal y exacta.

16. Es importante proponer a los estudiantes situaciones o problemas que les permitan generar y probar nuevas teorías.

17. El trabajo en solución de problemas retadores es una buena fuente para mostrar que cualquier persona puede hacer matemáticas.

18. Cuando un estudiante resuelve problemas lo importante es que sepa qué conceptos y procedimientos debe utilizar.

19. Los estudiantes se confunden si se les muestra más de una forma de resolver un mismo problema.

20. En la clase de matemáticas, el profesor debe saber la respuesta a cualquier pregunta de los estudiantes. 